\title{
Screening of potential biomarkers for chemoresistant ovarian carcinoma with miRNA expression profiling data by bioinformatics approach
}

\author{
SHIYANG WEI ${ }^{1}$, YAFENG WANG $^{2}, \mathrm{HONG}^{\mathrm{X}} \mathrm{W}^{1}$ and YAN KUANG ${ }^{1}$ \\ ${ }^{1}$ Department of Gynecology, The First Affiliated Hospital of Guangxi Medical University; ${ }^{2}$ Department of Anesthesiology, \\ People's Hospital of Guangxi Zhuang Autonomous Region, Nanning, Guangxi 530021, P.R. China
}

Received October 1, 2014; Accepted June 22, 2015

DOI: $10.3892 / 01.2015 .3610$

\begin{abstract}
The aim of the present study was to screen out the biomarkers associated with chemoresistance in ovarian carcinomas and to investigate the molecular mechanisms. microRNA (miRNA) expression data was obtained from published microarray data of the GSE43867 dataset from Gene Expression Omnibus (GEO), including the data of 86 chemotherapy-treated patients with serous epithelial ovarian carcinomas (response group, 36 complete response cases and 12 partial response cases; non-response group, 10 stable cases and 28 progressive disease cases), and identification of differentially-expressed miRNAs were conducted with a GEO2R online tool based on R language. TargetScan 6.2 was used to predict the targets of differentially-expressed miRNAs. Protein-protein interaction network analysis was conducted by STRING 9.1, while functional enrichment [Gene Ontology (GO) biological process terms] and Kyoto Encyclopedia of Genes and Genomes (KEGG) enrichment analyses were conducted by GeneCodis 3 for the target genes. A total of 6 differentially-expressed miRNAs were screened out, with 317 target genes obtained. It was found that 67 interactions existed among 76 genes/proteins through the PPI network analysis, and that 6 of these were potential key genes (PIK3R5, MAPK3, PTEN, S1PR3, BDKRB2 and NCBP2). The main biological processes involved in chemoresistant ovarian carcinoma were apoptosis, programmed cell death, cell migration, cell death and cell motility. The miRNA target genes were found to be associated with the ErbB signaling pathway, the gonadotropin-releasing hormone signaling pathway and other
\end{abstract}

Correspondence to: Professor Hong $\mathrm{Xu}$, Department of Gynecology, The First Affiliated Hospital of Guangxi Medical University, 6 Shuangyong Road, Nanning, Guangxi 530021, P.R. China

E-mail: nnxuhong@163.com

Key words: ovarian carcinomas, chemoresistance, microRNA, protein-protein interaction network, enrichment analysis pathways in cancer. IK3R5, MAPK3 and PIK3R5 are involved in the majority of GO terms and KEGG pathways associated with chemoresistance in ovarian carcinoma.

\section{Introduction}

Ovarian cancer is one of the leading causes of cancer incidence and cancer-associated mortality in women. Surgery combined with chemotherapy is the standard therapeutic strategy, and recurrence and chemoresistance in ovarian cancer patients are the main factors affecting the prognosis (1). Therefore, anti-resistance has become a prominent target for research into ovarian cancer $(2,3)$.

The combination data mining method increases the probability of identifying the biological processes and functional candidate genes that could represent the high-throughput data and have a great effect on the studied disease. A number of studies have revealed that applying in silico bioinformatic approaches in mining data from high-throughput microarray profiles is reliable and effective in predicting disease-causing biomarkers and has high accuracy (4,5). Qu et al (6) used microarray technology to profile microRNA (miRNA/miR) expression between CNE-2R and its parental cell line, CNE-2, and miR-205 was found to contribute to the radioresistance of nasopharyngeal carcinoma by directly targeting PTEN. In order to find feasible approaches for solving the chemoresistance in ovarian carcinoma, more and more studies have been performed in the last decade. Using microarray profiles, multiple potential biomarkers have been reported to be involved in chemoresistant ovarian carcinoma, including miR-106a, miR-591 (7), miR-23b, miR-27a (8), ARID1A (9) and Notch3 (10). However, the biological mechanisms of the biomarkers in chemoresistant ovarian carcinoma remain unclear.

The present study aimed to extract differentially-expressed miRNAs from microarray datasets from the Gene Expression Omnibus (GEO) database to probe their biological function in the development and progression of chemoresistant ovarian carcinoma. Information retrieved via miRNAs expression data, PPI interaction network construction and pathway enrichment analysis was combined to screen out potential biomarkers for chemoresistant ovarian carcinoma. This research will assist 
in disclosing the biomarkers of chemoresistance in ovarian carcinoma.

\section{Materials and methods}

miRNA expression profiles. The miRNA expression profile of the GSE43867 dataset was obtained from the GEO database (http://www.ncbi.nlm.nih.gov/geo/), which is based on the GPL16566 Applied Biosystems TaqMan Array Human miRNA A/B Cards v2.0 platform (Applied Biosystems Life Technologies, Foster City, CA, USA). This dataset included the miRNA profile expression microarrays from formalin-fixed and paraffin-embedded blocks of 86 chemotherapy-treated cases with serous epithelial ovarian carcinomas, which were submitted by Vecchione et al (11).

Screening of differentially-expressed miRNAs. GEO2R (http://www.ncbi.nlm.nih.gov/geo/geo2r/) is an interactive web tool that performs comparisons on original submitter-supplied processed data tables using the GEO query and limma $\mathrm{R}$ packages from the Bioconductor project (12). GEO2R was used to analyze the published microarray data of the GSE43867 dataset from the GEO database. In total, 86 chemotherapy-treated patients with serous epithelial ovarian carcinomas were divided into two groups: The response group consisted of 36 complete response cases and 12 partial response cases, while the non-response group consisted of 10 stable cases and 28 progressive disease cases. The results were downloaded in text format, and the miRNAs that met the cut-off criteria of $\mathrm{P}<0.05$ and a llog fold-changel of $>1.0$ were screened out as differentially-expressed miRNAs.

Prediction of target genes of differentially-expressed miRNAs. Targets of miRNAs are predicted by an online target prediction tool, TargetScan 6.2 (http://www.targetscan.org/) $(13,14)$, which predicts the biological targets of miRNAs by searching for the presence of conserved 8 mer and 7 mer sites that match the seed region of each miRNA. A prediction score of $>0.5$ is selected as a criterion for target genes with each miRNA.

Construction of a protein-protein interaction (PPI) network. STRING is a database of known and predicted PPIs based on the sources derived from the genomic context, high-throughput experiments, coexpression and previous knowledge (15). STRING quantitatively integrates interaction data from these sources for a large number of organisms, and transfers information between these organisms where applicable (16). The latest version, STRING9.1 (http://string-db.org/), covers 5,214,234 proteins from 1,133 organisms (17). In the present study, a PPI network of the miRNA target genes was constructed by STRING9.1, and highly-correlated genes/proteins (confidence score, $>0.7$ ) were selected as inclusion criteria for PPI network analysis.

Functional enrichment and pathway enrichment analysis. Functional enrichment (GO biological process terms) and Kyoto Encyclopedia of Genes and Genomes (KEGG) pathway enrichment analysis (KEGG and Panther pathways) were performed for the genes in the PPI network using the GeneCodis3 web tool (http://genecodis.dacya.ucm.es/) $(18,19)$. and
Table I. Differentially-expressed miRNAs obtained from the GSE43867 dataset.

\begin{tabular}{lll}
\hline miRNA & $\mid \operatorname{logFCl}$ & P-value \\
\hline hsa-miR-642 & 1.01956 & 0.00848 \\
hsa-miR-198 & 1.23491 & 0.01075 \\
hsa-miR-483-5p & 1.35478 & 0.01347 \\
hsa-miR-129-3p & 1.11770 & 0.02684 \\
hsa-miR-760 & 1.59264 & 0.03878 \\
hsa-miR-766 & 1.24657 & 0.03971 \\
\hline
\end{tabular}

miR/miRNA, microRNA; FC, fold-change.

the statistical test used for the enrichment was based on the hypergeometric distribution to compute P-values, which were corrected by the Benjamini and Hochberg false discovery rate method for multiple hypothesis testing $(\alpha=0.05)$. Only those terms with a value of $\mathrm{P}<0.05$ and a count number of $\geq 5$ genes were selected for analysis.

\section{Results}

Differentially-expressed miRNAs. A total of 6 differentially-expressed miRNAs were identified in the chemotherapy response cases compared with the non-response cases (control) in the serous epithelial ovarian carcinomas according to the criteria. The 6 miRNAs were hsa-miR-760, hsa-miR-483-5p, hsa-miR-766, hsa-miR-198, hsa-miR-129-3p and hsa-miR-642. The information for these 6 differentially-expressed miRNAs is presented in Table I.

Target genes and PPI network construction. A total of 317 target genes met the criteria of the 6 differentially-expressed miRNAs. The target genes were uploaded to the STRING online tool: A total of 67 interactions were found to exist among 76 proteins (genes) through the PPI network analysis, and 6 of them were potential key genes (PIK3R5, PTEN, MAPK3, S1PR3, BDKRB2 and NCBP2). Additionally, 37 proteins/genes were involved in the construction of the MAPK3 and NCBP2 module PPI network, as shown in Fig. 1.

GO term enrichment and KEGG analysis. The GeneCodis 3 online tool was used to characterize the biological functions of the genes that were included in the PPI network of the aforementioned potential key genes. Functional analysis demonstrated that the genes in this module could be enriched into 5 functional GO terms, as shown in Table II. The target genes were identified to be associated with GO categories such as regulation of transcription, apoptotic process and signal transduction, as shown in Table II. Based on KEGG pathway analysis, the target genes were found to be involved in the gonadotropin-releasing hormone signaling pathway, the ErbB signaling pathway and in other cancer pathways, as well as in the regulation of the actin cytoskeleton, as shown in Table III. 
Table II. Significant GO terms obtained from the microRNA targets in the protein-protein interaction network.

\begin{tabular}{lcc}
\hline GO term & \multicolumn{1}{c}{ Genes } \\
\hline $\begin{array}{l}\text { GO:0048011-nerve growth factor } \\
\text { receptor signaling pathway }\end{array}$ & MAPK3, APH1A, KALRN, PTEN, PREX1 \\
$\begin{array}{l}\text { GO:0045944-positive regulation of } \\
\text { transcription from RNA polymerase II promoter }\end{array}$ & NRIP1, MAPK3, PPARGC1A, MEF2D, STAT6, ETS1 \\
$\begin{array}{l}\text { GO:0045893-positive regulation of } \\
\text { transcription, DNA-dependent }\end{array}$ & NRIP1, MAPK3, PPARGC1A, ERBB4, ETS1 \\
$\begin{array}{l}\text { GO:0006915-apoptotic process } \\
\text { GO:0007165-signal transduction }\end{array}$ & APH1A, KALRN, PREX1, MEF2D, ERBB4 & 0.0008176 \\
\hline
\end{tabular}

GO, Gene Ontology.

Table III. KEGG pathways obtained from the miRNA targets.

\begin{tabular}{lll}
\hline Pathway & \multicolumn{1}{c}{ Genes } \\
\hline Kegg:04912 GnRH signaling pathway & MAPK3, MMP2, HBEGF, PLA2G5, GNRHR & 2.78x10-12 \\
Kegg:04012 ErbB signaling pathway & MAPK3, PIK3R5, ERBB4, HBEGF, CBL & 3.05x10 \\
Kegg:05200 Pathways in cancer & MAPK3, PIK3R5, MMP2, PTEN, ETS1, CBL & 3.21x10 \\
Kegg:04810 Regulation of actin cytoskeleton & MAPK3, PIK3R5, BDKRB2, PIP4K2B, IQGAP1 & 5.97x10 \\
\hline
\end{tabular}

GnRH, gonadotropin-releasing hormone.

\section{Discussion}

The present study identified 6 differentially-expressed miRNAs from the GEO GSE43867 dataset, which included a miRNA profile expression microarray for chemoresistant serous epithelial ovarian carcinomas, by bioinformatics analysis. In order to confirm the functions of these miRNAs, the target genes were predicted and the PPI network was constructed. Finally, functional annotations and pathway analysis were conducted to determine the biological mechanisms contributing to chemoresistant ovarian carcinoma.

The 6 differentially-expressed miRNAs were hsa-miR-760, hsa-miR-483-5p, hsa-miR-766, hsa-miR-198, hsa-miR-129-3p and hsa-miR-642. Iwaya et al (20) reported that miR-760 was downregulated in the bone marrow and primary tumor of advanced gastric cancer, while Wang et al (21) found that plasma miR-601 and miR-760 were of high value for discriminating advanced adenomas from normal controls. Zheng et al (22) found high expression levels of miR-483-5p in tumor tissues, plasma and cell lines of nasopharyngeal carcinoma patients. The results of a study by Wang et al (23) confirmed that miR-483-5p suppresses the proliferation of glioma cells via directly targeting extracellular signal-regulated kinase 1 (ERK1). The microarray results of a study by Yu et al (24) indicated that miR-483-5p was upregulated in ovarian serous carcinoma at stage III compared with stage I. miR-766 has been shown to be associated with esophageal cancer, cutaneous squamous cell carcinoma and lung adenocarcinoma (25-27), while miRNA-198 has been reported to be of high value for predicting the clinical outcomes of cancer $(28,29)$. Several studies have demonstrated the use of miR-129 as a diagnostic and prognostic biomarker for various tumors, such as renal cell carcinoma and gastric cancer $(30,31)$. It has also been reported that increasing miR-642 improves cisplatin sensitivity in advanced bladder cancer and cell lines (32).

In order to ascertain the function of the differentially-expressed miRNAs in the present study, the target genes of these miRNAs were retrieved by TargetScan 6.2, and 317 target genes were obtained. Through the construction of a PPI network of the target genes of 6 differentially expressed miRNAs, 76 genes/proteins were shown to be involved in the PPI network, and 6 of these were screened out as potential key genes for chemoresistant ovarian carcinoma. PIK3R5, PTEN, MAPK3, S1PR3, BDKRB2 and NCBP2 formed a module in the PPI network construction, indicating that the two genes, PIK3R5 and MAPK3, may play important roles in the development of chemoresistance in ovarian carcinoma. Results obtained from GO biological process and pathway enrichment analyses also lead to the identification of MAPK3 and PIK3R5 as key genes, which were involved in the majority of GO terms and KEGG pathways associated with chemoresistance in ovarian carcinoma.

MAPK3, also known as ERK1, is a member of the mitogen-activated protein kinase family. Amsterdam et al (33) reported that intense phosphorylated ERK1 and ERK2 was detected in the peripheral areas of stage II ovarian carcinoma. Jeong et al (34) found that ERK1/2 are important in docetaxel resistance in MCF-7 spheroids. In recent years, MAPK/ERK1 has been reported to be associated with various cancer types, and 


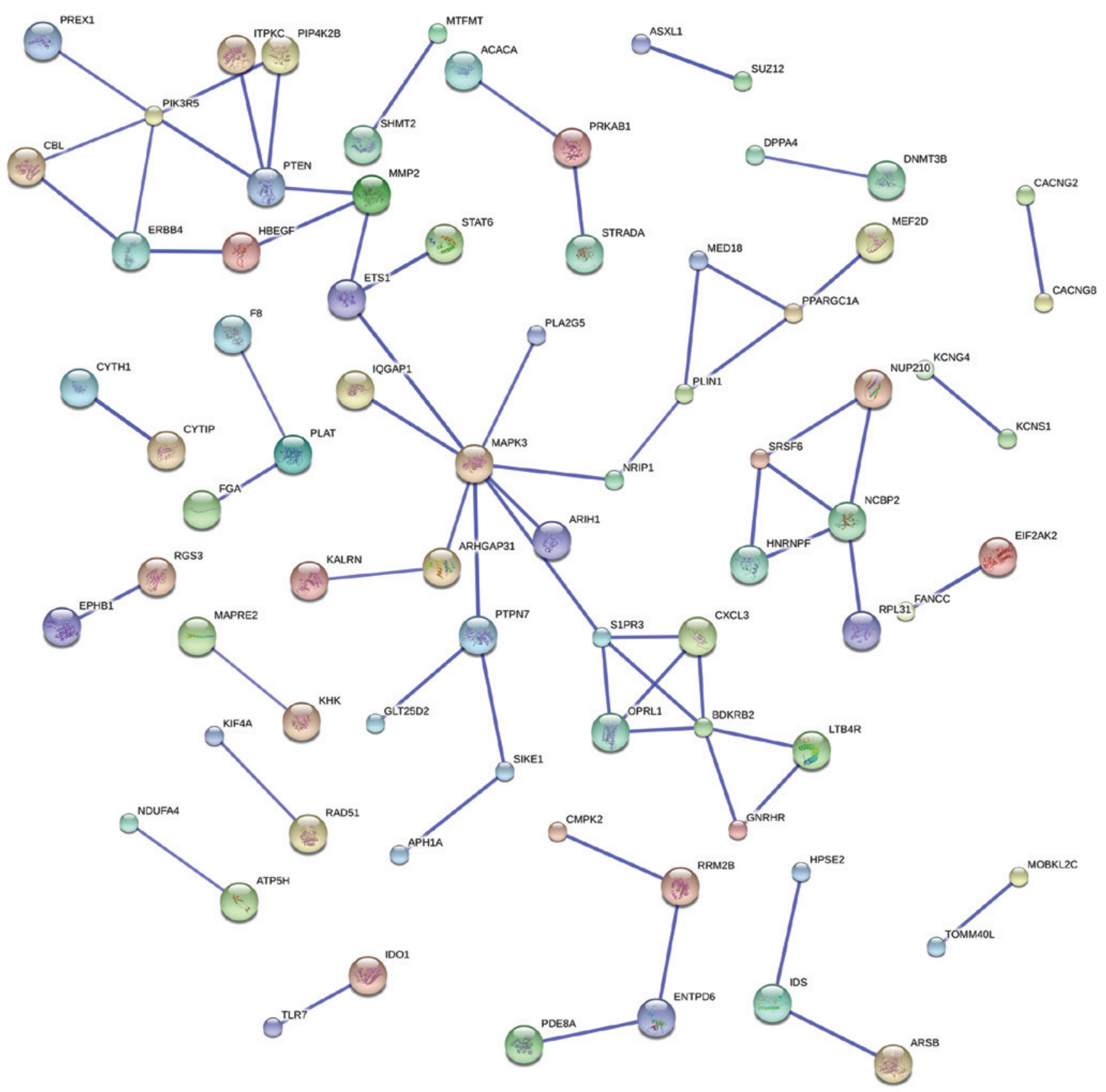

Figure 1. Protein-protein interaction network of microRNA genes constructed by STRING9.1.

it is believed that this gene participates in the process of cancer metastasis (35-37). PIK3R5 is a protein-coding gene. PIK3R5 has been reported to be associated with ataxia-oculomotor apraxia (38). Shull et al (39) reported that PIK3R5 mutations co-occurred with BRAF mutations, indicating that the gene may be a potential chemotherapeutic target for melanoma patients resistant to BRAF inhibitors. Through functional enrichment analysis, the present study identified the aforementioned pathways associated with the hub genes and their regulators. Therefore, MAPK3 and PIK3R5 may be selected as therapeutic targets for chemoresistant ovarian carcinoma. We speculate that MAPK3 and PIK3R5 may be associated with the occurrence and development of chemoresistant ovarian carcinoma.

In conclusion, a bioinformatic approach was applied to identify the differentially-expressed miRNAs in serous epithelial ovarian carcinomas samples of chemotherapy-treated responsive cases compared to those of non-responsive cases. The results suggested that MAPK3 and PIK3R5 may be associated with chemoresistant ovarian carcinoma. However, as of yet, there are no other studies to prove that MAPK3 and PIK3R5 are associated with chemoresistant ovarian carcinoma. The present study may provide novel insights into the molecular mechanism of chemoresistant ovarian carcinoma, thus, further studies regarding the association of the two genes and chemoresistant ovarian carcinoma are required.

\section{References}

1. Pignata S, Cannella L, Leopardo D, Pisano C, Bruni GS and Facchini G: Chemotherapy in epithelial ovarian cancer. Cancer Lett 303: 73-83, 2011. 
2. Khaider NG, Lane D, Matte I, Rancourt C and Piché A: Targeted ovarian cancer treatment: The TRAILs of resistance. Am J Cancer Res 2: 75-92, 2012

3. Kigawa J: New strategy for overcoming resistance to chemotherapy of ovarian cancer. Yonago Acta Med 56: 43-50, 2013.

4. Saei AA and Omidi Y: A glance at DNA microarray technology and applications. Bioimpacts 1: 75-86, 2011.

5. Cao WJ, Wu HL, He BS, Zhang YS and Zhang ZY: Analysis of long non-coding RNA expression profiles in gastric cancer. World J Gastroenterol 19: 3658-3664, 2013.

6. Qu C, Liang Z, Huang J, Zhao R, Su C, Wang S, Wang X, Zhang R, Lee MH and Yang H: MiR-205 determines the radioresistance of human nasopharyngeal carcinoma by directly targeting PTEN Cell Cycle 11: 785-796, 2012.

7. Huh JH, Kim TH, Kim K, Song JA, Jung YJ, Jeong JY, Lee MJ, Kim YK, Lee DH and An HJ: Dysregulation of miR-106a and miR-591 confers paclitaxel resistance to ovarian cancer. Br J Cancer 109: 452-461, 2013.

8. Park YT, Jeong JY, Lee MJ, Kim KI, Kim TH, Kwon YD, Lee C, Kim OJ and An HJ: MicroRNAs overexpressed in ovarian ALDH1-positive cells are associated with chemoresistance. J Ovarian Res 6: 18, 2013.

9. Katagiri A, Nakayama K, Rahman MT, Rahman M, Katagiri H, Nakayama N, Ishikawa M, Ishibashi T, Iida K, Kobayashi H, et al Loss of ARID1A expression is related to shorter progression-free survival and chemoresistance in ovarian clear cell carcinoma. Mod Pathol 25: 282-288, 2012.

10. Rahman MT, Nakayama K, Rahman M, Katagiri H, Katagiri A Ishibashi T, Ishikawa M, Iida K, Nakayama S, Otsuki Y and Miyazaki K: Notch3 overexpression as potential therapeutic target in advanced stage chemoresistant ovarian cancer. Am J Clin Pathol 138: 535-544, 2012

11. Vecchione A, Belletti B, Lovat F, Volinia S, Chiappetta G, Giglio S, Sonego M, Cirombella R, Onesti EC, Pellegrini P, et al: A microRNA signature defines chemoresistance in ovarian cancer through modulation of angiogenesis. Proc Natl Acad Sci USA 110: 9845-9850, 2013

12. Barrett T, Wilhite SE, Ledoux P, Evangelista C, Kim IF, Tomashevsky M, Marshall KA, Phillippy KH, Sherman PM, Holko M, et al: NCBI GEO: Archive for functional genomics data sets-update. Nucleic Acids Res 41: D991-D995, 2013.

13. Garcia DM, Baek D, Shin C, Bell GW, Grimson A and Bartel DP: Weak seed-pairing stability and high target-site abundance decrease the proficiency of lsy-6 and other microRNAs. Nat Struct Mol Biol 18: 1139-1146, 2011.

14. Grimson A, Farh KK, Johnston WK, Garrett-Engele P, Lim LP and Bartel DP: MicroRNA targeting specificity in mammals: Determinants beyond seed pairing. Mol Cell 27: 91-105, 2007.

15. Snel B, Lehmann G, Bork P and Huynen MA: STRING: A web-server to retrieve and display the repeatedly occurring neighbourhood of a gene. Nucleic Acids Res 28: 3442-3444, 2000

16. Szklarczyk D, Franceschini A, Kuhn M, Simonovic M, Roth A, Minguez P, Doerks T, Stark M, Muller J, Bork P, et al: The STRING database in 2011: Functional interaction networks of proteins, globally integrated and scored. Nucleic Acids Res 39: D561-D568, 2011.

17. Franceschini A, Szklarczyk D, Frankild S, Kuhn M, Simonovic M, Roth A, Lin J, Minguez P, Bork P, von Mering C and Jensen LJ: STRING v9.1: Protein-protein interaction networks, with increased coverage and integration. Nucleic Acids Res 41: D808-D815, 2013.

18. Tabas-Madrid D, Nogales-Cadenas R and Pascual-Montano A: GeneCodis3: A non-redundant and modular enrichment analysis tool for functional genomics. Nucleic Acids Res 40: W478-W483, 2012.

19. Nogales-Cadenas R, Carmona-Saez P, Vazquez M, Vicente C Yang X, Tirado F, Carazo JM and Pascual-Montano A: GeneCodis: Interpreting gene lists through enrichment analysis and integration of diverse biological information. Nucleic Acids Res 37: W317-W322, 2009.

20. Iwaya T, Fukagawa T, Suzuki Y, Takahashi Y, Sawada G, Ishibashi M, Kurashige J, Sudo T, Tanaka F, Shibata K, et al: Contrasting expression patterns of histone mRNA and microRNA 760 in patients with gastric cancer. Clin Cancer Res 19: 6438-6449, 2013.

21. Wang Q, Huang Z, Ni S, Xiao X, Xu Q, Wang L, Huang D, Tan C, Sheng W and Du X: Plasma miR-601 and miR-760 are novel biomarkers for the early detection of colorectal cancer. PLoS One 7: e44398, 2012
22. Zheng XH, Cui C, Ruan HL, Xue WQ, Zhang SD, Hu YZ, Zhou XX and Jia WH: Plasma microRNA profiles of nasopharyngeal carcinoma patients reveal miR-548q and miR-483-5p as potential biomarkers. Chin J Cancer 33: 330-338, 2014.

23. Wang L, Shi M, Hou S, Ding B, Liu L, Ji X, Zhang J and Deng Y: MiR-483-5p suppresses the proliferation of glioma cells via directly targeting ERK1. FEBS Lett 586: 1312-1317, 2012.

24. Yu X, Zhang X, Bi T, Ding Y, Zhao J, Wang C, Jia T, Han D, Guo G, Wang B, et al: MiRNA expression signature for potentially predicting the prognosis of ovarian serous carcinoma. Tumour Biol 34: 3501-3508, 2013.

25. Hummel R, Wang T, Watson DI, Michael MZ, Van der Hoek M, Haier $\mathbf{J}$ and Hussey DJ: Chemotherapy-induced modification of microRNA expression in esophageal cancer. Oncol Rep 26: 1011-1017, 2011

26. Li X, Shi Y, Yin Z, Xue X and Zhou B: An eight-miRNA signature as a potential biomarker for predicting survival in lung adenocarcinoma. J Transl Med 12: 159, 2014.

27. Sand M, Skrygan M, Georgas D, Sand D, Hahn SA, Gambichler T, Altmeyer P and Bechara FG: Microarray analysis of microRNA expression in cutaneous squamous cell carcinoma. J Dermatol Sci 68: 119-126, 2012.

28. Qi B, Yao WJ, Zhao BS, Qin XG, Wang Y, Wang WJ, Wang TY, Liu SG and Li HC: Involvement of microRNA-198 overexpression in the poor prognosis of esophageal cancer. Asian Pac J Cancer Prev 14: 5073-5076, 2013.

29. Han HS, Yun J, Lim SN, Han JH, Lee KH, Kim ST, Kang MH, Son SM, Lee YM, Choi SY, et al: Downregulation of cell-free miR-198 as a diagnostic biomarker for lung adenocarcinoma-associated malignant pleural effusion. Int J Cancer 133: 645-652, 2013.

30. Tsai KW, Wu CW, Hu LY, Li SC, Liao YL, Lai CH, Kao HW, Fang WL, Huang KH, Chan WC and Lin WC: Epigenetic regulation of miR-34b and miR-129 expression in gastric cancer. Int J Cancer 129: 2600-2610, 2011.

31. Chen X, Ruan A, Wang X, Han W, Wang R, Lou N, Ruan H, Qiu B, Yang H and Zhang X: miR-129-3p, as a diagnostic and prognostic biomarker for renal cell carcinoma, attenuates cell migration and invasion via downregulating multiple metastasis-related genes. J Cancer Res Clin Oncol 140: 1295-1304, 2014

32. Nordentoft I, Birkenkamp-Demtroder K, Agerbaek M, Theodorescu D, Ostenfeld MS, Hartmann A, Borre M, Ørntoft TF and Dyrskjøt L: miRNAs associated with chemo-sensitivity in cell lines and in advanced bladder cancer. BMC Med Genomics 5: 40, 2012

33. Amsterdam A, Shezen E, Raanan C, Schreiber L, Prus D, Slilat Y, Ben-Arie A and Seger R: Nuclear localization of phosphorylated ERK1 and ERK2 as markers for the progression of ovarian cancer. Int J Oncol 39: 649-656, 2011.

34. Jeong EK, Lee SY, Jeon HM, Ju MK, Kim CH and Kang HS: Role of extracellular signal-regulated kinase (ERK) $1 / 2$ in multicellular resistance to docetaxel in MCF-7 cells. Int J Oncol 37: 655-661, 2010

35. Guégan JP, Ezan F, Théret N, Langouët S and Baffet G: MAPK signaling in cisplatin-induced death: Predominant role of ERK1 over ERK2 in human hepatocellular carcinoma cells. Carcinogenesis 34: 38-47, 2013.

36. Zhang D, Li X, Yao Z, Wei C, Ning $\mathrm{N}$ and Li J: GABAergic signaling facilitates breast cancer metastasis by promoting ERK1/2-dependent phosphorylation. Cancer Lett 348: $100-108,2014$

37. Wu YY, Ma TL, Ge ZJ, Lin J, Ding WL, Feng JK, Zhou SJ, Chen GC, Tan YF and Cui GX: JWA gene regulates PANC-1 pancreatic cancer cell behaviors through MEK-ERK1/2 of the MAPK signaling pathway. Oncol Lett 8: 1859-1863, 2014.

38. Al Tassan N, Khalil D, Shinwari J, Al Sharif L, Bavi P, Abduljaleel Z, Abu Dhaim N, Magrashi A, Bobis S, Ahmed H, et al: A missense mutation in PIK3R5 gene in a family with ataxia and oculomotor apraxia. Hum Mutat 33: 351-354, 2012.

39. Shull AY, Latham-Schwark A, Ramasamy P, Leskoske K, Oroian D, Birtwistle MR and Buckhaults PJ: Novel somatic mutations to PI3 K pathway genes in metastatic melanoma. PLoS One 7: e43369, 2012. 